\title{
Overexpression of the ASPM gene is associated with aggressiveness and poor outcome in bladder cancer
}

\author{
ZHENGLIN XU ${ }^{1,2}$, QI ZHANG ${ }^{3}$, FRANK LUH ${ }^{4,5}$, BAIYE JIN ${ }^{1}$ and XIYONG LIU ${ }^{4,5}$ \\ ${ }^{1}$ Department of Urology, The First Affiliated Hospital, School of Medicine, Zhejiang University, Hangzhou, \\ Zhejiang 310006; ${ }^{2}$ Department of Urologic Surgery, Affiliated Dongyang People's Hospital of Wenzhou \\ Medical University, Dongyang, Zhejiang 322100; ${ }^{3}$ Department of Bioinformatics and Statistics, Hangzhou \\ Hope Biotechnology, Inc., Hangzhou, Zhejiang 310015, P.R. China; ${ }^{4}$ Sino-American Cancer Foundation \\ and ${ }^{5}$ Tumor Biomarker Development, California Cancer Institute, Temple City, CA 91780, USA
}

Received April 6, 2018; Accepted October 31, 2018

DOI: $10.3892 /$ ol.2018.9762

\begin{abstract}
Abnormal spindle-like microcephaly-associated $(A S P M)$ protein is essential for mitotic spindle function during cell replication. The present study aimed to evaluate the hypothesis that $A S P M$ serves a critical role in cancer invasiveness and may act as a prognostic biomarker in bladder cancer. In total, 6 independent worldwide bladder cancer microarray mRNA expression datasets $(n=1,355)$ with clinical and follow-up annotations were collected from the Gene Expression Omnibus (GEO) and The Cancer Genome Atlas (TCGA) databases. Reverse transcription-quantitative polymerase chain reaction analysis revealed that $A S P M$ mRNA expression was higher in bladder cancer tissue compared with adjacent normal bladder mucosae in 10 paired human tissue samples $(\mathrm{P}=0.004)$. ASPM overexpression in human bladder cancer samples was consistent with the mRNA expression datasets from GEO and TCGA. Bioinformatics analysis indicated that $A S P M$ mRNA expression was significantly
\end{abstract}

Correspondence to: Dr Baiye Jin, Department of Urology, The First Affiliated Hospital, School of Medicine, Zhejiang University, 79 Qinchun Raod, Hangzhou, Zhejiang 310006, P.R. China E-mail: 1189006@zju.edu.cn

Dr Xiyong Liu, Sino-American Cancer Foundation, California Cancer Institute, 4978 Santa Anita Ave, Suite 104, Temple City, CA 91780, USA

E-mail: xiyongliu@sacfamerica.org

Abbreviations: ASPM, abnormal spindle-like microcephalyassociated protein; MIBC, muscle-invasive bladder cancer; NMIBC, non-muscle-invasive bladder cancer; GEO, Gene Expression Omnibus; TCGA, The Cancer Genome Atlas; GSEA, gene set enrichment analysis; OS, overall survival; PFS, progression-free survival; HR, hazard ratio; 95\% CI, 95\% confidence interval; RCC, renal cell carcinoma; $\mathrm{PCa}$, prostate cancer

Key words: abnormal spindle-like microcephaly-associated protein, bladder cancer, prognostic biomarker, cancer survival, gene set enrichment analysis associated with grade and tumor node metastasis (TNM) stage in bladder cancer, based on pooled GEO and TCGA datasets $(\mathrm{P}<0.05)$. Stratification analysis indicated that the clinical significance of $A S P M$ was particularly pronounced in low-grade or papillary subtypes of bladder cancer. Individual Cox and pooled Kaplan-Meier analyses suggested that ASPM expression was significantly directly correlated with poor overall (OS) and progression-free survival (PFS) in bladder cancer. Multivariate and stratification analyses demonstrated that the prognostic significance of $A S P M$ was evident in low-grade or papillary bladder cancers, yet not in high-grade or non-papillary subgroups. Increased expression of ASPM was associated with poor OS in muscle-invasive bladder cancer and with poor PFS in non-muscle-invasive bladder cancer $(\mathrm{P}<0.05)$. Bioinformatics analysis identified the top 11 ASPM-related genes on STRING-DB.org. The expression of the majority of these genes was associated with poor outcomes of bladder cancer with statistical significance. Gene set enrichment analysis indicated that the high expression of ASPM could enrich gene signatures involved in mitosis, differentiation and metastasis in bladder cancer. Further analysis of TCGA datasets indicated that increased ASPM expression was significantly associated with higher Gleason score, $\mathrm{T}$ stage, $\mathrm{N}$ stage and poor clinical outcome in prostate cancer. It was also significantly associated with late TNM stage and poor PFS in renal cell carcinoma. In summary, ASPM may serve as a novel prognostic biomarker for low-grade or papillary bladder cancer.

\section{Introduction}

According to the American Cancer Society, 81,190 cases of bladder cancer (62,380 men and 18,810 women) will be diagnosed in the USA in 2018 (1). During the same year, $\sim 17,240$ bladder cancer-related deaths are expected to occur $(12,520$ men and 4,720 women) (1). Bladder cancer is the fourth most common cancer in men, but it is less common in women. In general, urothelial carcinoma (transitional cell carcinoma) accounts for $>90 \%$ of all bladder cancers, whereas squamous cell carcinoma accounts for $3-8 \%$ and adenocarcinoma for $1-2 \%$ of all cases. Approximately $75 \%$ of patients are diagnosed 
with non-muscle-invasive bladder cancer (NMIBC) (2), 50-70\% of whom develop disease recurrence and 10-15\% develop disease progression. Urinary tumor biomarkers, including $\mathrm{BTA}^{\circledR}, \mathrm{NMP}^{\circledR} 2^{\circledR}$, UroVysion ${ }^{\circledR}$ FISH and ImmunoCyt ${ }^{\mathrm{TM}}$, have been investigated for the purposes of improving the diagnosis, surveillance and staging of bladder cancer (3). However, their low specificity has limited the use of these biomarkers. Currently, standard guidelines recommend the use of these biomarkers as an adjunctive surveillance strategy, to reduce the need for invasive cystoscopy (3). Cxbladder is another urinary biomarker that measures the mRNA expression profile of five genes (IGF, HOXA, MDK, CDC and IL8R), with a sensitivity of $93 \%$ and specificity of $85 \%$. It has been used to classify highand low-risk subgroups of patients presenting with hematuria, to assess whether invasive cystoscopy is required (4).

Development of bladder cancer occurs via accumulation of molecular events. Genetic alterations may result in uncontrolled cellular proliferation, inhibition of differentiation and invasiveness of tumor cells. These changes determine tumor growth, relapse, progression and metastasis. It is necessary to identify reliable bladder cancer progression-associated genes in order to improve current methods of detecting and monitoring cancer recurrence and metastatic invasion.

Abnormal spindle-like microcephaly-associated (ASPM) gene, also referred to as abnormal spindle microtubule assembly, is located on chromosome 1q31 and encodes the ASPM protein. Expression of ASPM is essential for normal mitotic spindle function in embryonic neuroblasts and regulation of neurogenesis (5). Defects in the ASPM gene are associated with autosomal recessive primary microcephaly $(6,7)$. Neuronal depletion is associated with ASPM mutations and predominantly affects the anterior cortex during development $(8,9)$. An expression study revealed that $A S P M$ mRNA levels are higher in fetal tissues, but are very low in adult tissue (10). It has been reported that the ASPM gene is overexpressed in glioblastoma and malignant glioma compared with normal brain tissue (11-13). Knockdown of $A S P M$ by small interfering RNA was shown to reduce tumor cell and neural stem cell proliferation (12). ASPM expression is also associated with ependymoma recurrence in children (14). Abnormalities in ASPM expression are associated with numerous cancer types. $A S P M$ mRNA is overexpressed in hepatocellular carcinoma $(10,15)$. Upregulation is also observed in SV40 immortalized cells and non-small cell lung cancer tissues (16). It has also been proposed that upregulation of $A S P M$ expression increases the invasive capacity of melanoma cells (17). Studies in patients have revealed that $A S P M$ is significantly associated with poor outcomes in hepatocellular carcinoma (10), ovarian cancer (18), pancreatic cancer (19) and prostate cancer (20). However, to date, the clinical relevance and prognostic significance of $A S P M$ in bladder cancer remains unknown.

In the present study, the clinical relevance and prognostic significance of $A S P M$ were evaluated based on six bladder cancer gene expression datasets. Stratification and multivariate analyses were conducted to reduce confounding effects. Gene set enrichment analysis (GSEA) was performed to identify cancer-associated gene signatures of ASPM in bladder cancer. $A S P M$ co-expression proteins were also analyzed using the STRING database, and the prognostic relevance of the corresponding genes was evaluated. Based on these findings, the prognostic significance of $A S P M$ in bladder cancer was evaluated.

\section{Materials and methods}

Analysis of human bladder tissues by reverse transcription-quantitative polymerase chain reaction (RT-qPCR). Ten paired bladder cancer tissue samples ( 6 male and 4 female, age from 57 to 84) were collected from the Affiliated Dongyan People's Hospital, Wenzhou Medical University (Dongyang, China), according to a protocol approved by the Institutional Review Board. Informed consent was obtained from each participant. Total RNA from formalin-fixed paraffin-embedded tissue samples was extracted using an RN30-EASYspin kit (Aidab Biotechnologies Co., Ltd., Beijing, China) in order to evaluate ASPM mRNA expression. cDNA was produced from $1 \mu \mathrm{g}$ total RNA using the Promega M-MLV Reverse Transcriptase (Promega Corporation, Madison, WI, USA) and oligo dT primers according to the standard protocol. The ASPM primers for qPCR were as follows: Forward, 5'-AGC ATTCCTTTTATCCCAGAAACACCTG-3' and reverse, 5'-GCTTGCAGGGGATTTGTGATTTCTTCC-3'. Actin was used as a loading control. The human actin primers were as follows: Forward, 5'-CCCCAACTTGAGATGTATGAA GGCT-3' and reverse, 5'-TCTCAAGTCAGTGTACAGGTA AGCC 3'. RT-qPCR was performed using $1 \mu \mathrm{l}$ cDNA in a $50-\mu 1$ reaction volume. The thermocycling conditions were as follows: $95^{\circ} \mathrm{C}$ for $10 \mathrm{~min}$, followed by 40 cycles at $95^{\circ} \mathrm{C}$ for $10 \mathrm{sec}$ and at $60^{\circ} \mathrm{C}$ for $1 \mathrm{~min}$. The experiment was performed in triplicate.

Worldwide microarray gene expression datasets. A total of six independent worldwide bladder cancer microarray datasets were used in the present study. Four Gene Expression Omnibus (GEO) bladder cancer gene expression datasets were downloaded from www.ebi.ac.uk/arrayexpress; these datasets included GSE13507 (21), GSE31684 (22), E-MTAB-1803 (23) and E-MTAB-4321 (24). Two bladder cancer gene expression datasets, TCGA set1 and TCGA set2, were obtained from The Cancer Genomic Atlas (TCGA) research network: Cancergenome.nih.gov. All datasets contained clinical and follow-up annotations. Datasets without prognostic outcome information were excluded from the study. A total of 1,355 assessable bladder cancer cases with recurrence information and overall survival (OS) data were collected. Detailed information regarding the downloaded datasets is presented in Table I. Additional gene expression datasets of prostate cancer (PCa) $(n=496)$ and renal cell carcinoma (RCC) $(n=532)$ with clinical and outcome information were downloaded for further validating clinical meaning of ASPM.

Bioinformatics analysis. The detailed GSEA protocol can be obtained from the Broad Institute Gene Set Enrichment Analysis website (http://software.broadinstitute. org/gsea/index.jsp) (25). GSEA software v3.0 was used on a JAVA 8.0 platform. All dataset (.gct) and phenotype label (.cls) files were created and loaded into GSEA software, and gene sets were downloaded from the Broad Institute website. The number of permutations was set to 1,000 . A ranked-list metric 
Table I. Summary of downloaded gene expression data sets of bladder cancer.

\begin{tabular}{|c|c|c|c|c|c|c|}
\hline \multirow[b]{2}{*}{ Accession no. } & \multicolumn{4}{|c|}{ GEO } & \multicolumn{2}{|c|}{ TCGA } \\
\hline & GSE13507 & GSE31684 & E-MTAB-1803 & E-MTAB-4321 & TCGA set 1 & TCGA set 2 \\
\hline No. of patients & 256 & 93 & 170 & 476 & 407 & 131 \\
\hline Assessable cases ${ }^{\mathrm{a}}$ & 165 & 93 & 85 & 476 & 407 & 129 \\
\hline Date of study & NA & $1993-2004$ & NA & NA & NA & NA \\
\hline Platforms ${ }^{\mathrm{a}}$ & GPL6102 & GPL570 & NA & NA & NA & NA \\
\hline Country & South Korea & USA & NA & NA & NA & NA \\
\hline Sex & Y & $\mathrm{Y}$ & $\mathrm{Y}$ & $\mathrm{Y}$ & NA & $\mathrm{Y}$ \\
\hline Age at diagnosis & $66(24-88)$ & $69(41-91)$ & $69(44-89)$ & $69(24-95)$ & $68(34-90)$ & $69(34-88)$ \\
\hline Grade & $\mathrm{Y}$ & $\mathrm{Y}$ & $\mathrm{Y}$ & $\mathrm{Y}$ & $\mathrm{Y}$ & $\mathrm{Y}$ \\
\hline Tumor size & NA & NA & NA & $\mathrm{Y}$ & NA & NA \\
\hline TNM stage & $\mathrm{Y}$ & NA & NA & NA & $\mathrm{Y}$ & $\mathrm{Y}$ \\
\hline Tumor stage & $\mathrm{Y}$ & $\mathrm{Y}$ & $\mathrm{Y}$ & $\mathrm{Y}$ & Y & $\mathrm{Y}$ \\
\hline Lymph node & $\mathrm{Y}$ & $\mathrm{Y}$ & $\mathrm{Y}$ & NA & Y & $\mathrm{Y}$ \\
\hline Metastasis & $\mathrm{Y}$ & $\mathrm{Y}$ & $\mathrm{Y}$ & NA & $\mathrm{Y}$ & $\mathrm{Y}$ \\
\hline Tobacco smoking history & NA & $\mathrm{Y}$ & NA & NA & NA & Y \\
\hline OS months (range) & $1.03-136.97$ & $0.39-175.5$ & $0-132$ & NA & $0-165.9$ & $0-140.7$ \\
\hline Recurrence months (range) & NA & $0.39-175.5$ & NA & NA & NA & $2.76-43.97$ \\
\hline Progression months (range) & NA & NA & NA & $0-74.9$ & $0-163.2$ & NA \\
\hline
\end{tabular}

aPlatforms: GPL6102, Illumina human-6 v2.0 expression bead chip; GPL570, [HG-U133_Plus_2] Affymetrix Human Genome U133 Plus 2.0 Array. GEO, Gene expression omnibus; TCGA, the cancer genome atlas; OS, overall survival TNM, tumor node metastasis. Y, yes information is available; NA, information is not available; GSE, Gene Expression Omnibus Series format; E-MTAB, experiment in MicroArray Gene Expression Tabular format.

was generated by calculating the signal-to-noise ratio, which is based on the difference of means scaled according to the standard deviation.

Data management. Each database was downloaded, converted, constructed and managed using Microsoft Excel (version 2007, Microsoft Corporation, Redmond, WA, USA) and Python software (www.python.org). For pooled analysis, the expression levels of genes were normalized prior to merging. ASPM and related genes were re-stratified into four grades $(\mathrm{Q} 1, \mathrm{Q} 2$, Q3 and Q4), based on the percentiles of each independently downloaded dataset. The Q1 subgroup ( $<25 \%$ percentile) was considered as a reference for statistical analysis. Subgroups Q2, Q3 and Q4 were $\geq 25$ to $50 \%,>50$ to $<75 \%$, and $\geq 75 \%$ respectively. In addition, expression levels less than the median value were designated 'ASPM-low', and levels greater than or equal to the median value were designated 'ASPM-high'.

Statistical analysis. R software (www.r-project.org) and JMP 10.0 software (SAS Institute, Cary, NC, USA) were used for general statistical analysis. Group comparisons of continuous data were performed using paired t-tests for independent means or one-way analyses of variance with Tukey's post-hoc test for multiple groups. Categorical variables were compared using $\chi^{2}$ analysis, Fisher's exact test or the binomial test of proportions. Kaplan-Meier analysis and Cox proportional hazard models were used to analyze OS and progression-free survival (PFS). In PFS analysis, patients with distant metastatic bladder cancer not completely resected were excluded. Multivariate Cox analysis was applied to adjust for covariate effects, and stratification analysis was used to reduce the confounding effect of the calculated hazard ratios (HRs). Missing data were coded and excluded from the analysis.

\section{Results}

Association of ASPM $m$ RNA expression with bladder cancer invasiveness. The mRNA expression levels of ASPM in normal bladder mucosae and bladder cancer tissue samples were determined by RT-qPCR. In the 10 paired samples, the mRNA expression of $A S P M$ was significantly increased in primary bladder cancer compared with adjacent normal bladder mucosae ( $\mathrm{P}=0.004$; Fig. 1A). Further bioinformatics data from the GSE13507 dataset supported this finding. As indicated in Fig. 1B, ASPM mRNA levels in primary bladder cancer and recurrent NMIBC were significantly higher compared with normal bladder, as well as normal bladder mucosae adjacent to cancer tissue $(\mathrm{P}<0.01)$.

The clinical relevance of $A S P M$ was further investigated in six gene expression datasets, comprising a total of 1,355 bladder cancer cases. The median ASPM expression level was used as a cut-off point for each dataset. All participants were stratified as $A S P M$-high or ASPM-low and then merged. As indicated in Table II, analysis revealed that the percentage of ASPM-high was significantly and positively associated with high-grade bladder cancer, lymph node involvement and smoking history 
Table II. Demographic distribution of ASPM in bladder cancer.

\begin{tabular}{|c|c|c|c|c|c|c|}
\hline \multirow[b]{2}{*}{ Characteristics } & \multicolumn{3}{|c|}{ GEO } & \multicolumn{3}{|c|}{ TCGA } \\
\hline & High (\%) & Low (\%) & P-values ${ }^{a}$ & High (\%) & Low (\%) & P-values ${ }^{\mathrm{a}}$ \\
\hline \multicolumn{7}{|l|}{ Sex } \\
\hline Male & 319 (49.8) & $321(50.2)$ & & $48(49.5)$ & $49(50.5)$ & \\
\hline Female & $92(51.4)$ & 87 (48.6) & 0.713 & 17(53.1) & $15(46.9)$ & 0.721 \\
\hline \multicolumn{7}{|l|}{ Age } \\
\hline$<55$ yrs. & $40(40.8)$ & $58(59.2)$ & & $25(45.5)$ & $30(54.5)$ & \\
\hline$\geq 55$ yrs. & $371(51.5)$ & $350(48.5)$ & 0.048 & $245(50.9)$ & $236(49.1)$ & 0.441 \\
\hline \multicolumn{7}{|l|}{ Tumor size } \\
\hline$<3 \mathrm{~cm}$ & $125(44.2)$ & $158(55.8)$ & & & & \\
\hline$\geq 3 \mathrm{~cm}$ & $60(69.0)$ & 27 (31.0) & $<0.001$ & & & \\
\hline \multicolumn{7}{|l|}{ Grade } \\
\hline Low & $276(65.1)$ & 148 (34.9) & & $1(3.70)$ & $26(96.3)$ & \\
\hline High & $133(34.3)$ & 255 (65.7) & $<0.001$ & 267 (52.9) & $238(47.1)$ & $<0.001$ \\
\hline \multicolumn{7}{|l|}{ TNM stage } \\
\hline 0 & $3(13.0)$ & $20(87.0)$ & & & & \\
\hline I & $35(43.8)$ & $45(56.2)$ & & $2(66.7)$ & $1(33.3)$ & \\
\hline II & $16(61.5)$ & $10(38.5)$ & & $47(43.1)$ & $62(56.9)$ & \\
\hline III & $15(75.0)$ & $5(25.0)$ & & $64(54.7)$ & $53(45.3)$ & \\
\hline IV & $13(81.2)$ & $3(18.8)$ & $<0.001$ & $61(57.0)$ & $46(43.0)$ & 0.163 \\
\hline \multicolumn{7}{|l|}{ Subtype } \\
\hline NMIBC & 266 (45.9) & $313(54.1)$ & & $1(100.0)$ & $0(0.00)$ & \\
\hline MIBC & 145 (60.4) & 95 (39.6) & $<0.001$ & $262(50.9)$ & 253 (49.1) & 0.326 \\
\hline \multicolumn{7}{|l|}{ Tumor stage } \\
\hline $\mathrm{Ta}$ & 148 (39.5) & $226(60.4)$ & & & & \\
\hline $\mathrm{T} 1$ & 115 (56.9) & $87(43.1)$ & & $3(75.0)$ & $1(25.0)$ & \\
\hline $\mathrm{T} 2$ & $46(58.2)$ & $33(41.8)$ & & $67(45.0)$ & $82(55.0)$ & \\
\hline $\mathrm{T} 3$ & $54(56.2)$ & $42(43.8)$ & & 138 (53.7) & $119(46.3)$ & \\
\hline $\mathrm{T} 4$ & $31(62.3)$ & $18(36.7)$ & $<0.001$ & 35 (46.7) & $40(53.3)$ & 0.236 \\
\hline \multicolumn{7}{|l|}{ Node stage } \\
\hline N0 & $110(45.5)$ & $132(54.5)$ & & $150(48.4)$ & $160(51.6)$ & \\
\hline NX & $10(58.8)$ & 7 (41.2) & & $25(54.4)$ & $21(45.6)$ & \\
\hline N1 & $38(58.5)$ & $27(41.5)$ & & $36(63.2)$ & $21(36.8)$ & \\
\hline $\mathrm{N} 2$ & $6(100.0)$ & $0(0.0)$ & & $44(44.0)$ & $56(56.0)$ & \\
\hline N3 & $1(100.0)$ & $0(0.0)$ & 0.023 & $11(78.6)$ & $3(21.4)$ & 0.032 \\
\hline \multicolumn{7}{|l|}{ Metastasis } \\
\hline M0 & $78(49.4)$ & $80(50.6)$ & & $131(48.3)$ & $140(51.7)$ & \\
\hline M1 & $4(57.1)$ & $3(42.9)$ & 0.687 & 7 (46.7) & $8(53.3)$ & 0.568 \\
\hline \multicolumn{7}{|l|}{ Smoking status } \\
\hline Never & $6(33.3)$ & $12(66.7)$ & & $55(39.0)$ & $86(61.0)$ & \\
\hline Yes & $90(53.6)$ & $78(46.4)$ & 0.084 & $203(53.7)$ & $175(46.3)$ & 0.003 \\
\hline
\end{tabular}

There are $819,819,370,812,819,819,800,331,165,93,342,819,103$ and 84 cases of Sex, Age, Tumor size, Grade, TNM stage, Subtype, Tumor stage, Node stage, Metastasis stage, Smoking status, Chemotherapy, Cytechomy, Intravesical therapy, Radiotherapy in pooled GEO dataset. There are 129, 536, 532, 336, 516, 485, 527, 532, 519, 260 and 274 cases of Sex, Age, Grade, TNM stage, Subtype, Tumor stage, Node stage, Metastasis stage, Smoking status, Chemotherapy, Radiotherapy in pooled TCGA dataset. P-values was based on Pearson Chi-square test.

in the pooled GEO and TCGA datasets $(\mathrm{P}<0.05)$. ASPM expression was also significantly associated with tumor stage and TNM stage in the pooled GEO dataset $(\mathrm{P}<0.05)$ but not in the TCGA dataset $(\mathrm{P}>0.05)$. ASPM expression was 
A

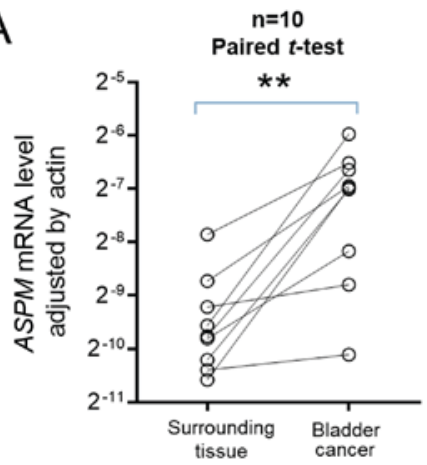

C

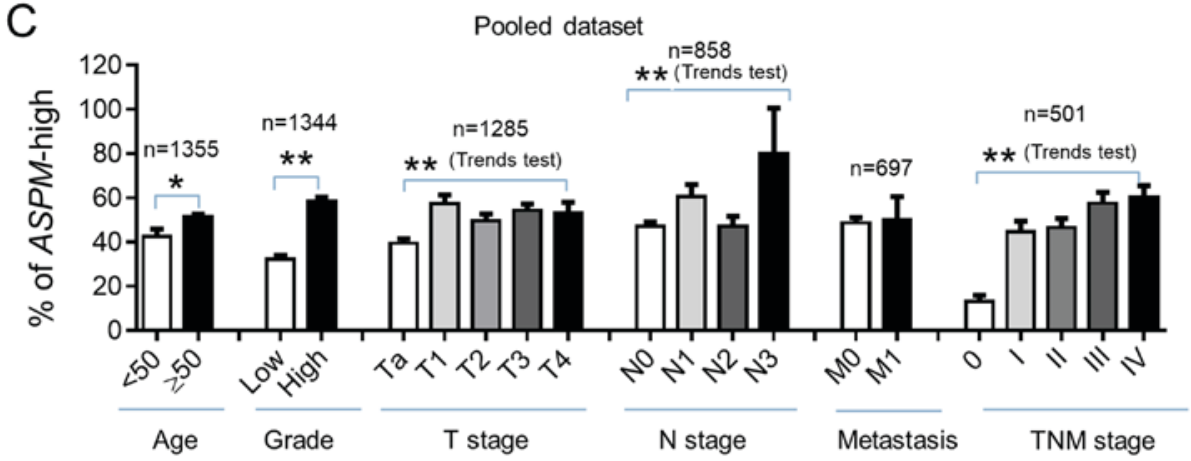

D

High-grade (pooled dataset)

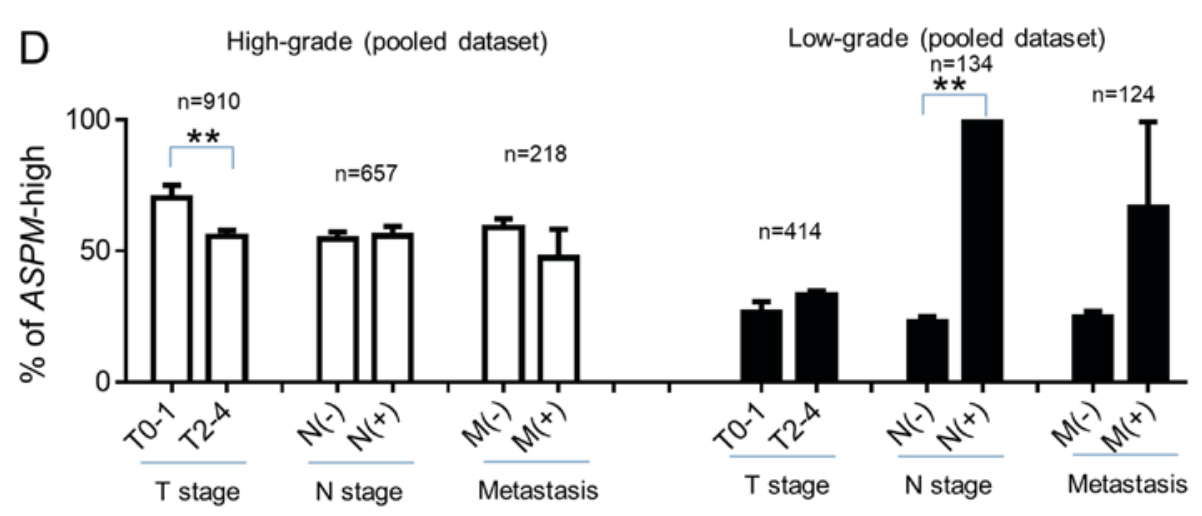

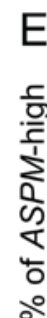

B

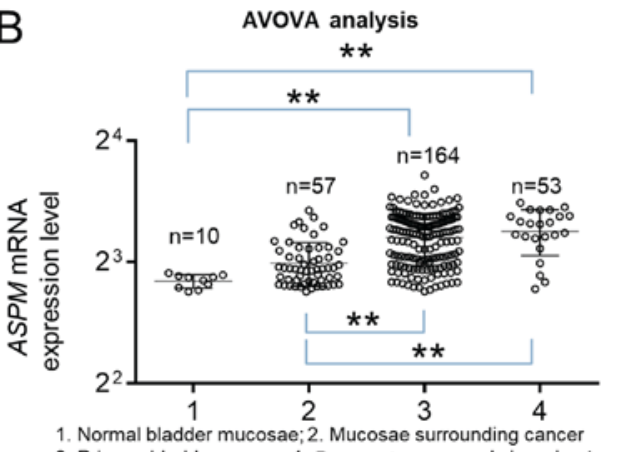

3. Primary bladder cancer; 4. Recurrent non-muscle invasion tumor

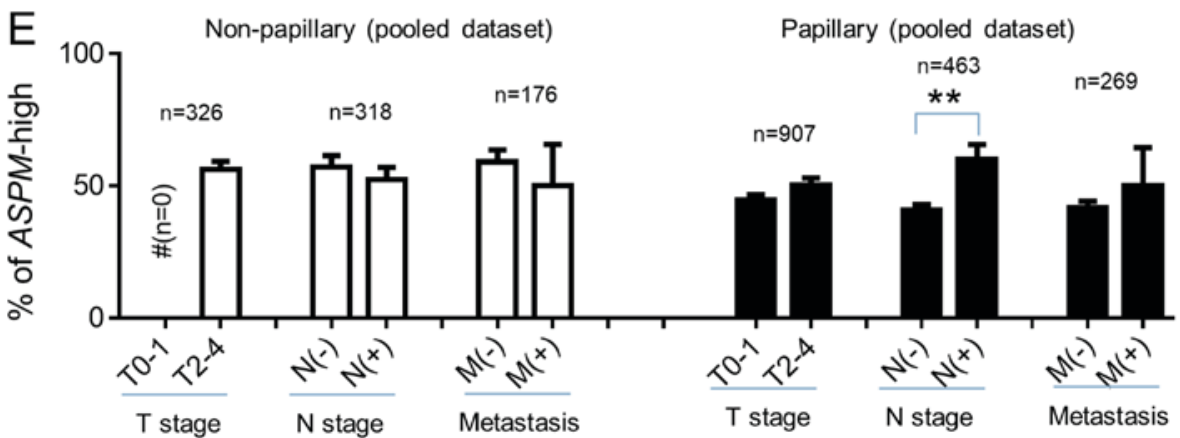

Figure 1. ASPM mRNA expression and clinical characteristics of bladder carcinoma. The mRNA expression of ASPM in bladder cancer tissue and adjacent bladder mucosae was determined by RT-qPCR analysis. The difference in mRNA levels between normal and bladder cancer tissues was consistent with the GSE13507 dataset. In the pooled dataset, bladder cancer cases were designated as either ASPM-high or ASPM-low according to the median expression value in each dataset. (A) Relative $A S P M$ mRNA expression in primary bladder cancer and adjacent normal bladder mucosae. Actin was used as an internal reference. (B) Comparison of ASPM mRNA expression in normal bladder mucosae, mucosae adjacent to bladder cancer, primary bladder cancer and recurrent non-muscle invasive bladder cancer in the GSE13507 dataset. (C) Distribution of ASPM mRNA expression according to clinical characteristics, including age, grade, tumor stage, lymph node involvement, distant metastasis and TNM stage. (D) ASPM distribution and TNM stage in high- and low-grade bladder cancers . (E) Clinical relevance of ASPM in papillary and non-papillary bladder cancer histological subtypes. \#, statistical test could not be performed due to there being no T0-1 stage in non-papillary bladder cancer. ASPM, abnormal spindle-like microcephaly-associated protein; RT-qPCR, reverse transcription-quantitative polymerase chain reaction. ${ }^{*} \mathrm{P}<0.05 ;{ }^{* *} \mathrm{P}<0.01$.

significantly associated with grade, tumor stage and lymph node involvement in the overall pooled dataset (GEO + TCGA; Fig. 1C). In addition, analysis indicated that ASPM expression was more likely to be significantly associated with lymph node involvement and metastasis in low-grade (Fig. 1D) and papillary (Fig. 1E) bladder cancer. T0-1 stage bladder cancer was 
A

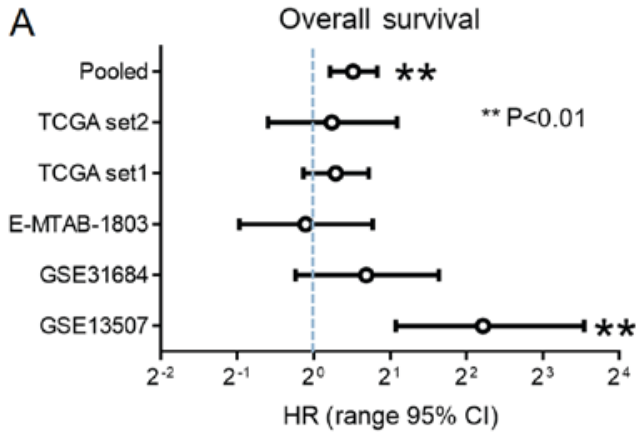

C

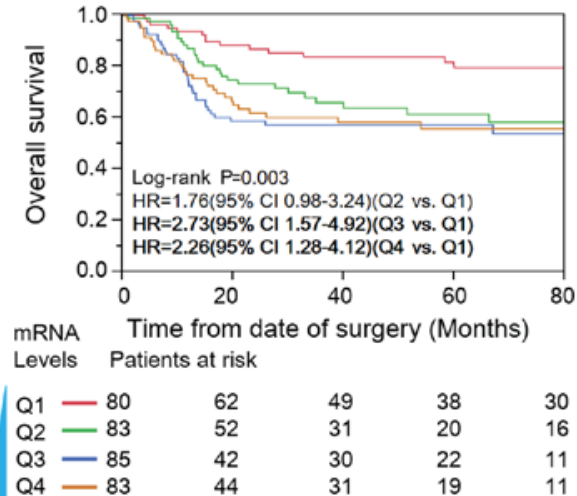

E

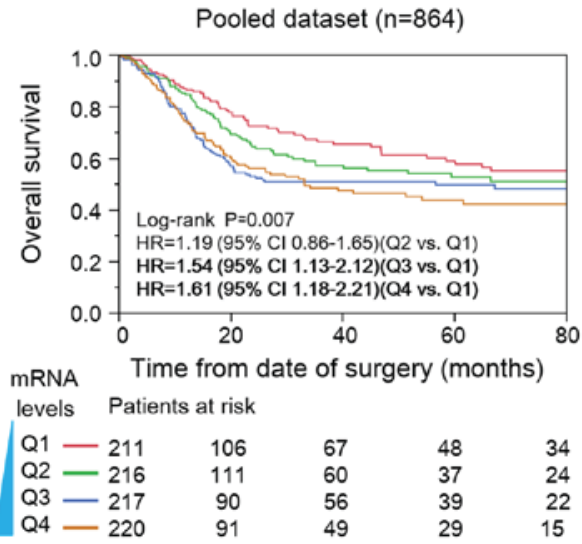

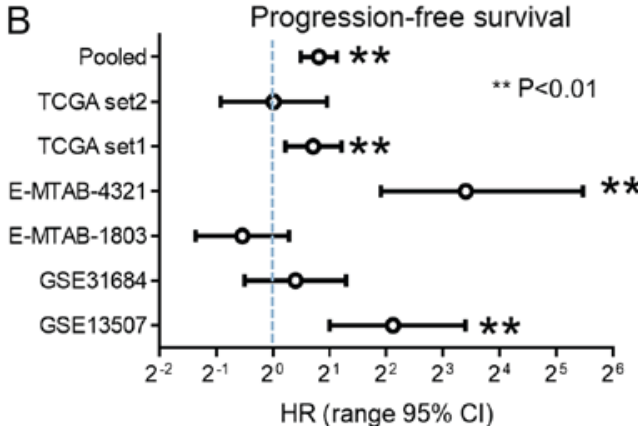

D

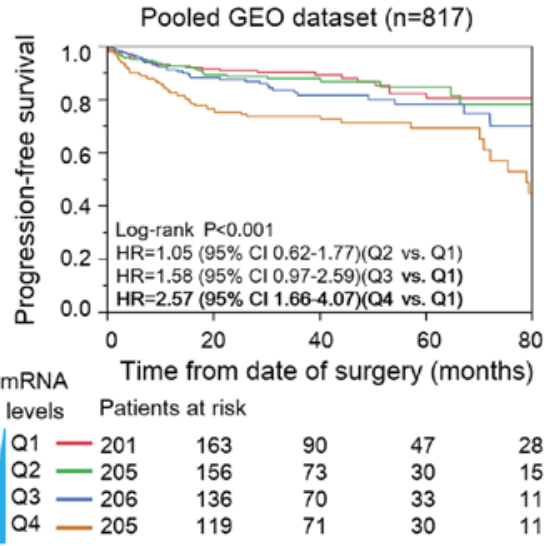

$\mathrm{F}$

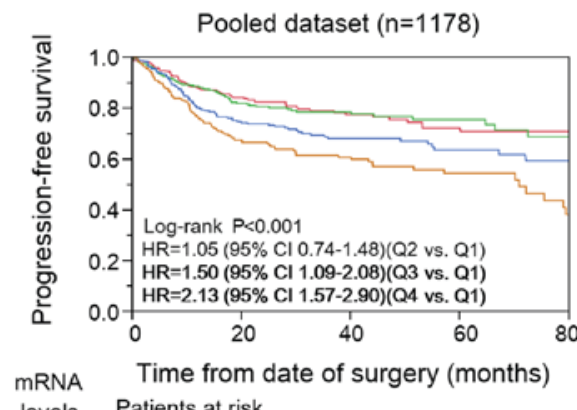

Patients at risk

$\begin{array}{lllll}\text { Q1 }-293 & 190 & 99 & 53 & 30 \\ \text { Q2 }-289 & 197 & 97 & 44 & 22 \\ \text { Q3 - 299 } & 163 & 89 & 44 & 19 \\ \text { Q4 } 297 & 147 & 80 & 35 & 14\end{array}$

Figure 2. Cox proportional hazard and Kaplan-Meier analysis of ASPM and outcome in bladder cancer datasets. (A) Cox analysis of each dataset. HR represents the relative risk of death from bladder cancer (ASPM-high vs. ASPM-low). Pooled HR was measured for all participants. Age and sex were used to adjust for HR. (B) Age- and sex-adjusted HR of disease progression was determined in each bladder cancer dataset. (C) In Kaplan-Meier analysis, bladder cancers were stratified into four subgroups (Q1, Q2, Q3 and Q4), based on the quartile values of each dataset. The Kaplan-Meier plot demonstrates the percentage of overall survival for each bladder cancer subgroup in the pooled GEO dataset. (D) Kaplan-Meier analysis for progression-free survival and expression levels of ASPM in the pooled GEO dataset. (E) Overall survival was analyzed in the pooled in GEO + TCGA dataset. (F) Progression-free survival analysis for pooled GEO and TCGA datasets. ASPM, abnormal spindle-like microcephaly-associated protein; HR, hazard ratio; GEO, Gene Expression Omnibus; TCGA, The Cancer Genome Atlas. ${ }^{* *} \mathrm{P}<0.01$.

considered to be NMIBC, and T2-4 stage was considered to be muscle-invasive bladder cancer (MIBC). ASPM expression was weakly associated with MIBC (T2-4 stage) in low-grade and papillary bladder cancer, but this result was not identified as statistically significant. However, ASPM expression was significantly increased in high-grade NMIBC $(\mathrm{P}<0.001)$. Since there is no T0-1 stage non-papillary bladder cancer, the association between ASPM and T stage could not be evaluated in this subgroup. Further stratification analysis indicated that $A S P M$ was also significantly associated with high grade in both NMIBC and MIBC $(\mathrm{P}<0.001)$. An association between ASPM and aggressive characteristics was observed in both male and female bladder cancer patients. Overall, gene expression data suggested that $A S P M$ was significantly associated with invasive pathological characteristics in bladder cancer, particularly in low-grade and papillary bladder cancer subtypes.

Prognostic significance of ASPM in bladder cancer. The aforementioned findings suggested that $A S P M$ may be associated with poor survival in bladder cancer. Cox proportional hazard and Kaplan-Meier analyses were performed to evaluate the prognostic significance of $A S P M$ in bladder cancer. As 
A

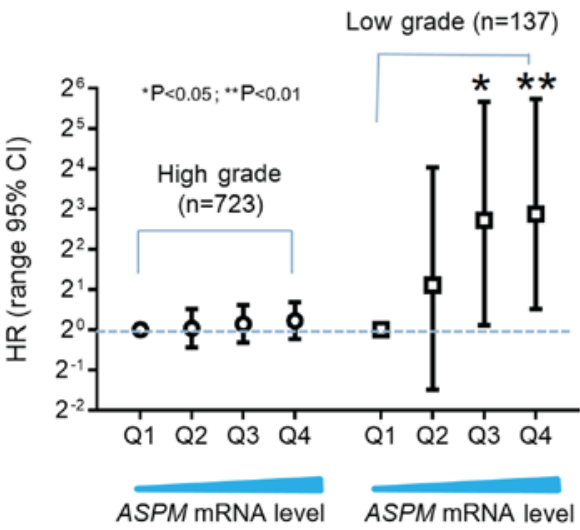

C

Pooled dataset

Overall survival

Papillary $(n=168)$

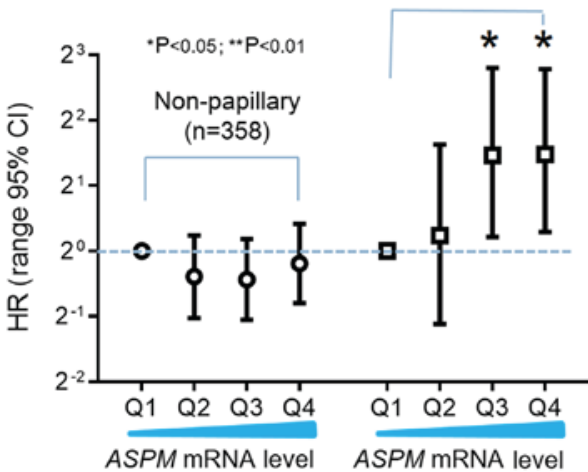

E

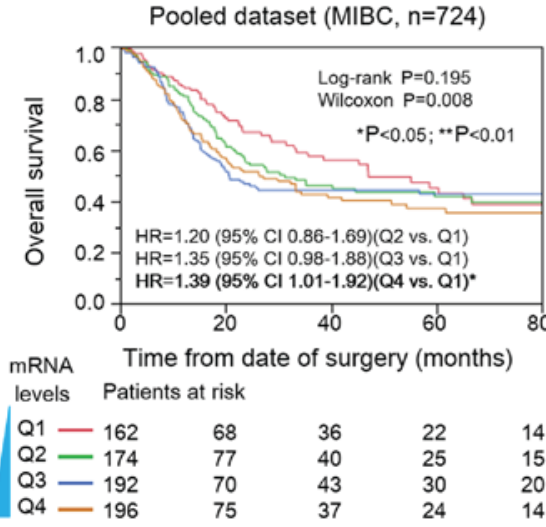

B

Pooled dataset

Progression-free surviva

Low grade $(n=409)$

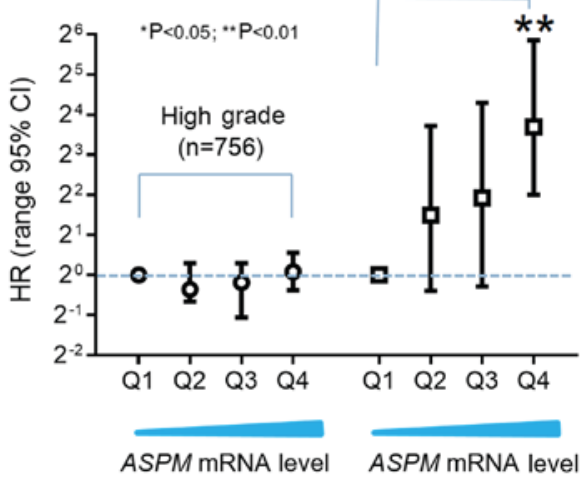

D

Pooled datase

Progression-free survival

Papillary $(n=533)$

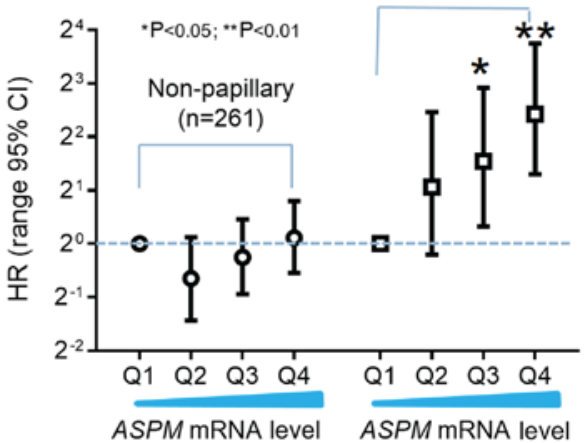

F

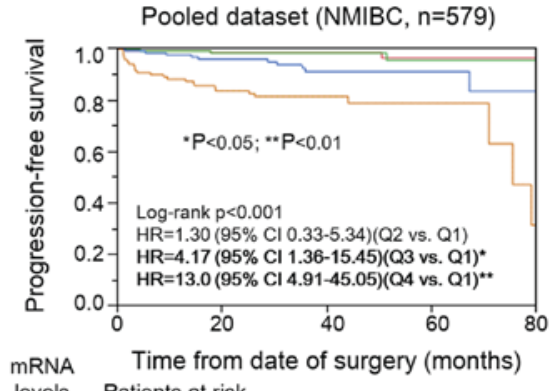

levels Patients at risk

\begin{tabular}{|lllll}
$\mathrm{Q} 1-158$ & 142 & 77 & 38 & 22 \\
$\mathrm{Q} 2-155$ & 103 & 62 & 22 & 12 \\
$\mathrm{Q} 3-140$ & 115 & 55 & 23 & 5 \\
$\mathrm{Q} 4-126$ & 88 & 50 & 17 & 3
\end{tabular}

Figure 3. Stratification analysis for ASPM mRNA expression and outcome in bladder cancer. The overall survival analysis result is indicated in the left column and progression-free survival is indicated in the right column. In the upper row, the prognostic significance of $A S P M$ was evaluated in high-grade and low-grade bladder cancers. Cox analysis of (A) overall survival and (B) progression-free survival. The middle row represents (C) overall survival and (D) disease-free survival (D) in papillary and non-papillary bladder cancers. The lower row represents the prognostic significance of $A S P M$ in (E) MIBC and (F) NMIBC subgroups. ASPM, abnormal spindle-like microcephaly-associated protein; MIBC, muscle-invasive bladder cancer; NMIBC, non-muscle-invasive bladder cancer.

indicated in Fig. 2A, Cox analysis revealed ASPM-high was significantly associated with relative risk of death from bladder cancer in the GSE13507 dataset $(\mathrm{P}<0.01)$. In pooled (GEO+TCGA) analysis, ASPM was significantly associated with poor OS. The HR was 1.46 (95\% CI: 1.14-1.78; $\mathrm{P}<0.01$ ). In the TCGA dataset1, E-MTAB-4321 datasets and GSE13507 datasets, $A S P M$ expression was significantly associated with progression of bladder cancer $(\mathrm{P}<0.01)$. The pooled HR of $A S P M$ for PFS was 1.76 (95\% CI: 1.41-2.19; P<0.01; Fig. 2B).
Furthermore, Kaplan-Meier plots revealed that $A S P M$ was significantly associated with poor OS and PFS in the pooled GEO dataset (Fig. 2C and D) and the overall pooled dataset (Fig. 2E and F).

Prognostic significance of ASPM expression in bladder cancer subtypes. To reduce potential confounding effects, further stratification and multivariate analysis were conducted to investigate the prognostic significance of ASPM expression 
A

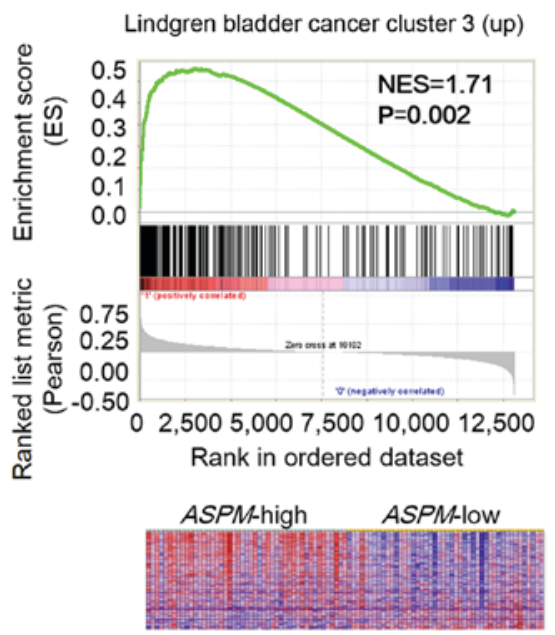

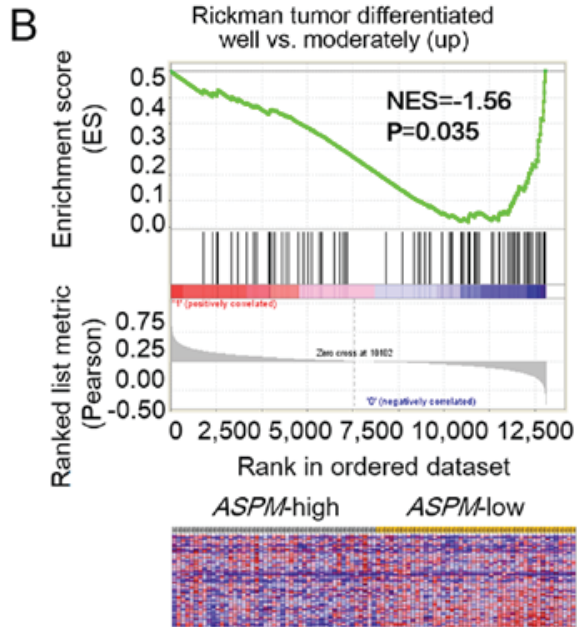

${ }^{*} P<0.05,{ }^{* *} P<0.01$

C

Gene signatures

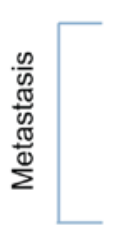

ALONSO_METASTASIS_UP JAEGER_METASTASIS_UP. SUNG_METASTASIS_STROMA_DN TOMIDA_METASTASIS_UP. BIDUS_METASTASIS_UP. JIMETASTASIS_REPRESSED_BY_STK11. RIZ_ERYTHROID_DIFFERENTIATION_CCNE1RHODES_UNDIFFERENTIATED_CANCERLE_NEURONAL_DIFFERENTIATION_DNZHAN_VARIABLE_EARLY_DIFFERENTIATION_GENES_DN-
RIZ_ERYTHROID_DIFFERENTIATION RICKMAN_TUMOR_DIFFERENTIATED_WELL_VS_MODERATELY_UP. RIZ_ERYTHROID_DIFFERENTIATION_HEMGNInvasion $\square$ WANG_TUMOR_INVASIVENESS_UPPUIFFE_INVASION_INHIBITED_BY_ASCITES_UP. - HERNANDEZ_MITOTIC_ARREST_BY_DOCETAXEL_1_DN Mitosis REICHERT_MITOSIS_LIN9_TARGETS-

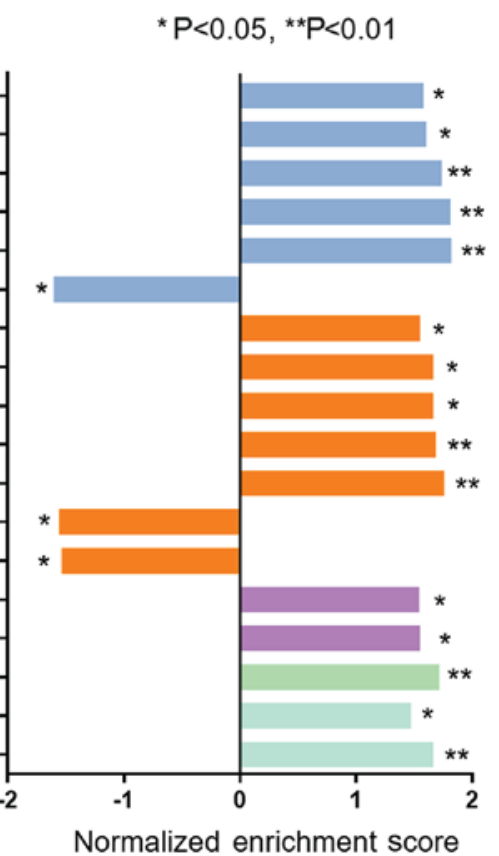

Figure 4. Analysis for gene sets enriched by ASPM in bladder carcinoma. Gene set enrichment analysis was used to identify gene signatures (sets) enriched by $A S P M$ expression. (A) Gene signature of Lindgren bladder cancer cluster 3 was enriched by high expression of ASPM. (B) Low expression of ASPM enriched Rickman tumor differentiation well vs. moderately gene signature. (C) List of cancer-related gene signatures (sets) with statistical significance. ASPM, abnormal spindle-like microcephaly-associated protein. ${ }^{*} \mathrm{P}<0.05 ;{ }^{* *} \mathrm{P}<0.01$.

in bladder cancer subtypes. Aforementioned findings indicated that $A S P M$ was associated with invasive characteristics in low-grade rather than high-grade bladder cancer (Fig. 1D). Stratification analysis indicated that increased ASPM expression significantly promoted relative progression in low-grade bladder cancer, and was directly correlated with the risk of death (Fig. 3A and B). Notably, the number of cases in the high-grade subtype was notably higher compared with the low-grade subtype in both the OS and PFS survival analyses, which rules out the possibility that any statistical significance could be caused by a larger sample size. Cox analysis revealed that $A S P M$ expression was associated with OS and PFS in the papillary histological subtype, but not in the non-papillary subtype (Fig 3C and D), which was consistent with the aforementioned findings (Fig. 1E). The prognostic significance of ASPM in MIBC and NMBC was also determined (Fig. 3E and F). Kaplan-Meier plots revealed that higher ASPM expression was associated with poor OS in MIBC (Wilcoxon $\mathrm{P}=0.008$; Fig. 3E), and $A S P M$ was significantly associated with poor PFS in NMIBC (log-rank $\mathrm{P}<0.01$; Fig. $3 \mathrm{~F}$ ). These findings suggest that $A S P M$ may serve as a prognostic biomarker for low-grade or papillary bladder cancer.

ASPM-associated genes and their prognostic significance in bladder cancer. GSEA was performed to investigate the association between ASPM gene expression and cancer-related gene signatures (Fig. 4). A heat map revealed that the majority of genes in the Lindgren bladder cancer cluster 3 (up) signature were upregulated (labeled red) in the ASPM-high subgroup (Fig. 4A), whereas genes in the Rickman tumor differentiated 
A

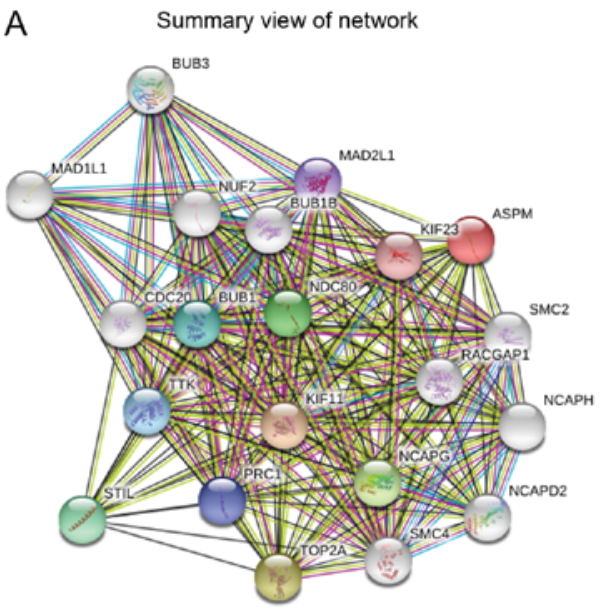

C
B

\begin{tabular}{clc}
\multicolumn{3}{c}{ Summary view of Network of ASPM in homo sapiens } \\
\hline Gene symbol & Annotation & Score* \\
\hline KIF11 & Kinesin Family Member 11 & 0.974 \\
TOP2A & DNA Topoisomerase II Alpha & 0.937 \\
NCAPG & Non-SMC Condensin I Complex Subunit G & 0.936 \\
NDC80 & NDC80, Kinetochore Complex Component & 0.934 \\
STIL & STIL, Centriolar Assembly Protein & 0.933 \\
BUB1 & BUB1 Mitotic Checkpoint Serine/Threonine Kinase & 0.926 \\
TTK & TTK Protein Kinase & 0.926 \\
PRC1 & Protein Regulator Of Cytokinesis 1 & 0.916 \\
MAD2L1 & Mitotic Arrest Deficient 2 Like 1 & 0.915 \\
KIF23 & Kinesin Family Member 23 & 0.912 \\
SMC4 & Structural Maintenance Of Chromosomes 4 & 0.904 \\
\hline "Score is computed by combining the probebillies from the different evidence channels and corrected \\
for the probablity of randomly observing an interaction.
\end{tabular}

PFS (Pooled dataset, $n=1178$ )
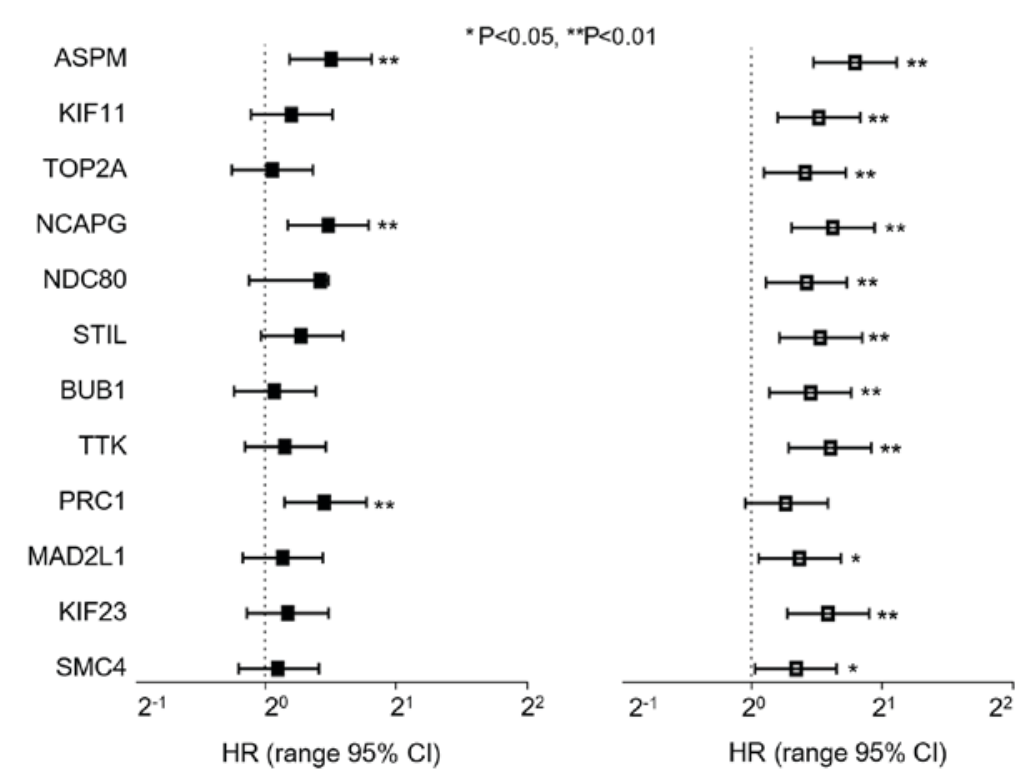

Figure 5. ASPM-related proteins and their prognostic significance in bladder cancer. (A) Data on ASPM-interacting proteins were obtained from STRING (string-db.org). (B) Annotation of ASPM interacting proteins and their co-expression scores. (C) Cox proportional hazard analysis for ASPM-related genes in the pooled bladder cancer dataset. ASPM, abnormal spindle-like microcephaly-associated protein. $\mathrm{P}<0.05 ;{ }^{* *} \mathrm{P}<0.01$.

well vs. moderately (up) signature were enriched in the $A S P M$-low subgroup (Fig. 4B). The gene signatures enriched by $A S P M$ are presented in Fig. 4C. ASPM-high enriched signatures included: Metastasis [BIDUS Metastasis (UP) and TOMIDA Metastasis (UP)], differentiation [RHODES Undifferentiation Cancer and LE Neuronal Differentiation (DN)], invasion [WANG Tumor Invasiveness (UP)], mitosis (REICHERT Mitosis) and bladder cancer (Fig. 4C). By contrast, RIZ Erythroid Differentiation HEMGN and JI Metastasis Repressed by STK11 were enriched in the ASPM-low subgroup (Fig. 4C).

$A S P M$ network proteins were identified by STRING (string-db.org) and are presented in Fig. 5A. The top 11 proteins and corresponding gene names, annotations and scores are listed in Fig. 5B. These genes included: KIF11, TOP2A, NCAPG, NDC80, STIL, BUB1, TTK, PRC1, MAD2L1, KIF23 and SMC4. These genes are related to cell mitosis, DNA replication and cell cycle checkpoint. This was consistent with the findings from GSEA. Subsequently, the prognostic significance of the mRNA expression levels was analyzed. As indicated in Fig. 5C, Cox analysis indicated that the majority of these genes had $\mathrm{HR}$ values $>1$. In addition, PFS analysis revealed that all the genes except PRC1 were significantly associated with progression of bladder cancer $(\mathrm{P}<0.05)$. Overall, these findings indicate that $A S P M$ is involved with bladder cancer invasiveness through interactions with several genes related to cell proliferation, undifferentiation and the metastasis signaling pathway.

The clinical significance of ASPM in prostate cancer and renal cell carcinoma. The clinical relevance and prognostic significance of $A S P M$ was also validated in urologic cancers, including prostate cancer $(\mathrm{PCa}$ ) and renal cell carcinoma ( $\mathrm{RCC}$ ) (Fig. 6). The expression of ASPM was found to be associated 

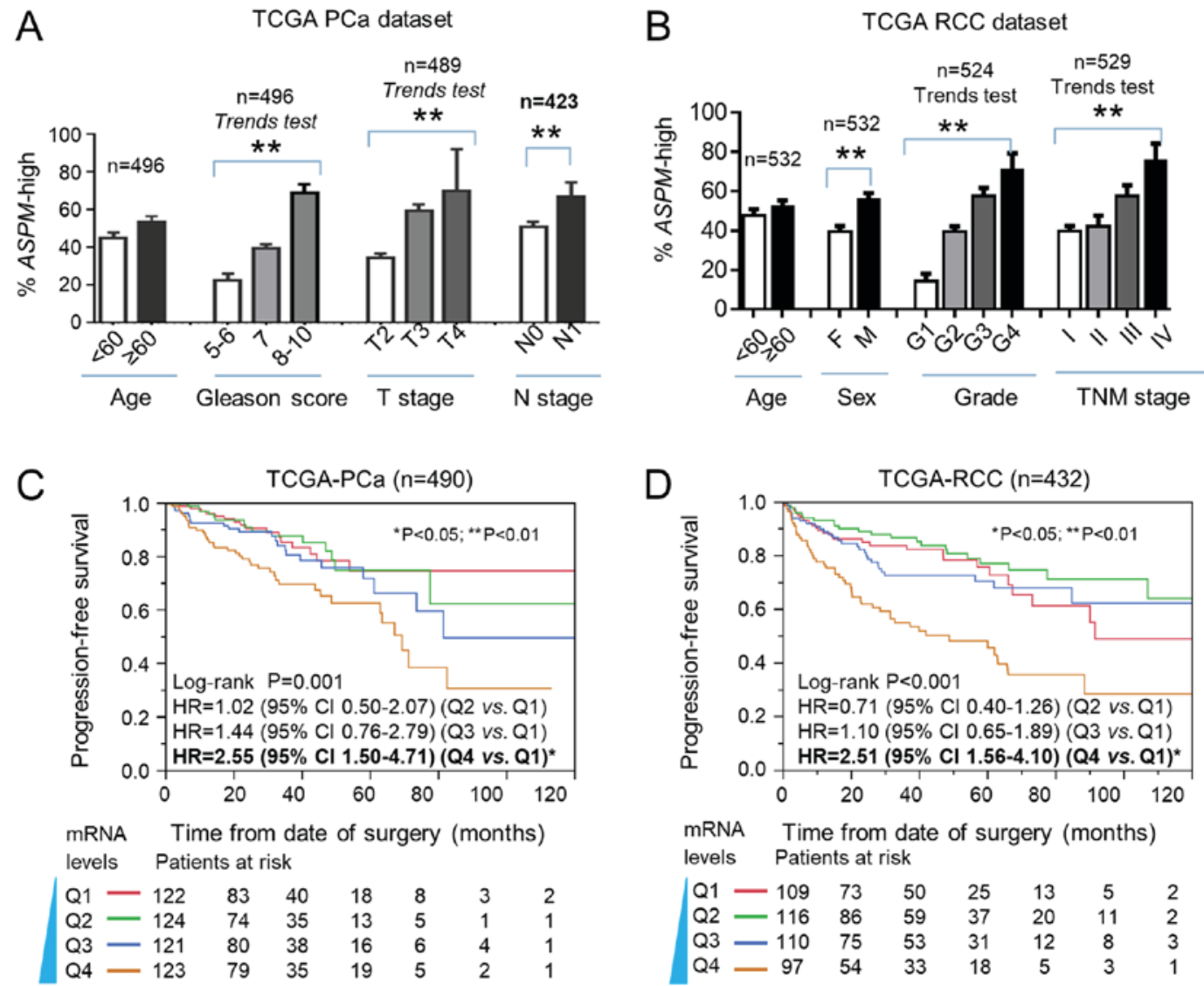

Figure 6. Clinical relevance and prognostic significance of ASPM in RCC and PCa. High-throughput PCa and RCC gene expression data were obtained from TCGA. (A) TCGA ASPM mRNA expression and clinical characteristics in PCa. (B) Clinical relevance of ASPM in RCC. (C) Kaplan-Meier and Cox analysis of $A S P M$ and outcome in PCa. (D) Prognostic significance of ASPM mRNA expression in RCC. ASPM, abnormal spindle-like microcephaly-associated protein; RCC, renal cell carcinoma; PCa, prostate cancer. " $\mathrm{P}<0.05$; * $\mathrm{P}<0.01$.

with Gleason score, $\mathrm{T}$ stage and $\mathrm{N}$ stage of $\mathrm{PCa}(\mathrm{P}<0.05$; Fig. 6A). ASPM expression was also significantly associated with older age and later TNM stage in RCC (Fig. 6B). Kaplan-Meier and Cox analysis revealed that ASPM mRNA expression was significantly associated with PFS in PCa and RCC, based on TCGA datasets ( $\mathrm{P}<0.01$; Fig. $6 \mathrm{C}$ and $\mathrm{D})$.

\section{Discussion}

In the present study, it was demonstrated that $A S P M$ was significantly overexpressed in bladder cancer and was associated with invasive pathological characteristics, including high grade and advanced TNM stage in bladder cancer, based on the GEO and TCGA datasets. In the pooled analysis, $A S P M$ was significantly associated with poor OS and PFS in a dose-dependent manner. In order to verify this result, stratification and multivariate analyses were conducted to reduce potential confounding effects. It was indicated that the clinical and prognostic significance of $A S P M$ was particularly pronounced in the low-grade and papillary bladder cancer subtypes. The prognostic significance of $A S P M$ was also examined in six bladder cancer gene datasets. Individual analysis results revealed that the prognostic performance of $A S P M$ for each dataset varied. The percentage of low-grade and histological subtypes of bladder cancer in each dataset was different, which explains the variation in prognostic performance in each dataset. In addition, it was also observed that $A S P M$ could predict PFS in NMIBC and OS in MIBC. In the present study, participant data were collected from several countries with different socioeconomic backgrounds, suggesting that the prognostic significance of $A S P M$ is universal. Overall, our current findings suggest that $A S P M$ may serve as a prognostic biomarker for bladder cancer of the low-grade and papillary histological subtypes. ASPM may also serve as a potential therapeutic biomarker for bladder cancer treatment.

Our analysis further validated that $A S P M$ was associated with progression and poor outcomes of other two urologic cancers, including prostate cancer (PCa) and renal cell carcinoma (RCC) (Fig. 6). An association between ASPM mRNA expression and biochemical recurrence in $\mathrm{PCa}$ has been previously reported (20). Kaplan-Meier analysis of ASPM mRNA in liver, endometrial, pancreatic and lung cancer was obtained from the Human Protein Atlas (www.proteinatlas. org/ENSG00000066279-ASPM/pathology). Furthermore, prognostic significance of ASPM was also reported in hepatocellular carcinoma (10), ovarian cancer (18) and pancreatic cancer (19). These findings suggest that $A S P M$ may serve as a prognostic biomarker for a number of other cancer types, in addition to bladder cancer.

The mechanism by which ASPM promotes low-grade bladder cancer aggressiveness is not yet known. In the present study, the top 11 proteins in the ASPM network were examined (Fig. 5). The functions of these genes are implicated in cell mitosis, DNA synthesis, DNA three-dimensional folding and 
cell proliferation. The GSEA results indicated that $A S P M$ could enrich mitosis, differentiation and metastasis gene signatures in bladder cancer (Fig. 4). Previous studies have reported that ASPM maintains the migratory and pro-metastatic potential of cancer stem cells (CSCs) and multiple glandular cancers, including pancreatic, breast and prostate cancer (19). ASPM may augment canonical and non-canonical Wnt signaling by positively regulating disheveled or other upstream Wnt signaling components to activate Wnt signaling and cancer aggressiveness in subpopulations of CSCs. Further studies are required in order to elucidate the mechanism underlying the role of ASPM in cancer cell division, undifferentiation and invasion.

A limitation of the present study is a lack of immunohistochemistry (IHC) data to support the conclusions. The prognostic significance of ASPM protein expression for bladder cancer was not evaluated due to non-specific staining on IHC analysis (data not shown). The Human Protein Atlas validated the ASPM antibody and also concluded that ASPM IHC results were inconsistent with mRNA expression data (www.proteinatlas.org/ENSG00000066279-ASPM/antibody). To date, data on ASPM protein expression and outcomes in cancer have not been reported due to the poor performance of ASPM antibodies on IHC staining. In the present study, RT-qPCR was used to replace IHC in order to evaluate differences in expression between normal and bladder cancer samples. Another limitation of this study is that therapeutic significance of ASPM could not be evaluated due to insufficient chemotherapy and radiotherapy information. The prognostic and predictive performance of $A S P M$ will be investigated further in our future studies.

In summary, the present study investigated the clinical significance and prognostic value of $A S P M$ mRNA expression in bladder cancer based on six gene expression datasets. It was concluded that $A S P M$ expression is significantly associated with bladder cancer aggressiveness in the low-grade and papillary histological subtypes. Therefore, ASPM appears to be a promising prognostic biomarker and potential therapeutic target in bladder cancer.

\section{Acknowledgements}

The authors would like to thank NIH Gene Expression Omnibus, ArrayExpress (www.ebi.ac.uk/arrayexpress) and The Cancer Genome Atlas pilot platform (established by NCI and NHGRI; cancergenome.nih.gov), which made the genomic and clinical bladder cancer data available.

\section{Funding}

This project was supported by the Key Project of the Science and Technology Program of Zhejiang Province (grant no. 2014C03028), Donyang Hospital Research Supporting Program and the Sino-America Cancer Foundation.

\section{Availability of data and materials}

The datasets used and/or analyzed during the current study are available from the corresponding author on reasonable request.

\section{Authors' contributions}

ZX collected data and interpreted the results of data analysis. QZ performed statistical and bioinformatics analysis for bladder cancer datasets. FL provided scientific suggestions on study design and English editing of the manuscript. BJ designed the study. XL analyzed the data, and was a major contributor to writing the manuscript. All authors have read and approved the final version of this manuscript for publication.

\section{Ethics approval and consent to participate}

Ten paired bladder cancer tissue samples were collected from the Affiliated Dongyan People's Hospital, Wenzhou Medical University (Dongyang, China), according to a protocol approved by the Institutional Review Board. Informed consent was obtained from each participant. All other high-throughput gene expression data and clinical information of bladder cancer were obtained from public genomic databases. All identifying information had been removed from downloaded datasets. Our research meets the publication guidelines provided by TCGA (cancergenome.nih.gov/publications/publicationguidelines).

\section{Patient consent for publication}

All identifying information had been removed. Informed consent was obtained from each participant.

\section{Competing interests}

The authors declare that they have no competing interests.

\section{References}

1. Siegel RL, Miller KD and Jemal A: Cancer statistics, 2018. CA Cancer J Clin 68: 7-30, 2018.

2. Babjuk M, Burger M, Zigeuner R, Shariat SF, van Rhijn BW, Compérat E, Sylvester RJ, Kaasinen E, Böhle A, Palou Redorta J, et al: EAU guidelines on non-muscle-invasive urothelial carcinoma of the bladder: Update 2013. Eur Urol 64: 639-653, 2013.

3. Velázquez N: Bladder cancer academy 2018 selected summaries. Rev Urol 20: 31-37, 2018.

4. Darling D, Luxmanan C, O'Sullivan P, Lough T and Suttie J: Clinical utility of cxbladder for the diagnosis of urothelial carcinoma. Adv Ther 34: 1087-1096, 2017.

5. Kouprina N, Pavlicek A, Collins NK, Nakano M, Noskov VN, Ohzeki J, Mochida GH, Risinger JI, Goldsmith P, Gunsior M, et al: The microcephaly ASPM gene is expressed in proliferating tissues and encodes for a mitotic spindle protein. Hum Mol Genet 14: 2155-2165, 2005.

6. Kaindl AM, Passemard S, Kumar P, Kraemer N, Issa L, Zwirner A, Gerard B, Verloes A, Mani S and Gressens P: Many roads lead to primary autosomal recessive microcephaly. Prog Neurobiol 90: 363-383, 2010.

7. Higgins J, Midgley C, Bergh AM, Bell SM, Askham JM, Roberts E, Binns RK, Sharif SM, Bennett C, Glover DM, et al: Human ASPM participates in spindle organisation, spindle orientation and cytokinesis. BMC Cell Biol 11: 85, 2010.

8. Desir J, Cassart M, David P, Van Bogaert P and Abramowicz M: Primary microcephaly with ASPM mutation shows simplified cortical gyration with antero-posterior gradient pre- and post-natally. Am J Med Genet A 146A: 1439-1443, 2008.

9. Kouprina N, Pavlicek A, Mochida GH, Solomon G, Gersch W, Yoon YH, Collura R, Ruvolo M, Barrett JC, Woods CG, et al: Accelerated evolution of the ASPM gene controlling brain size begins prior to human brain expansion. PLoS Biol 2: E126, 2004. 
10. Lin SY, Pan HW, Liu SH, Jeng YM, Hu FC, Peng SY, Lai PL and Hsu HC: ASPM is a novel marker for vascular invasion, early recurrence, and poor prognosis of hepatocellular carcinoma. Clin Cancer Res 14: 4814-4820, 2008.

11. Hagemann C, Anacker J, Gerngras S, Kühnel S, Said HM, Patel R, Kämmerer U, Vordermark D, Roosen K and Vince GH: Expression analysis of the autosomal recessive primary microcephaly genes MCPH1 (microcephalin) and MCPH5 (ASPM, abnormal spindle-like, microcephaly associated) in human malignant gliomas. Oncol Rep 20: 301-308, 2008.

12. Horvath S, Zhang B, Carlson M, Lu KV, Zhu S, Felciano RM, Laurance MF, Zhao W, Qi S, Chen Z, et al: Analysis of oncogenic signaling networks in glioblastoma identifies ASPM as a molecular target. Proc Natl Acad Sci USA 103: 17402-17407, 2006.

13. Bikeye SN, Colin C, Marie Y, Vampouille R, Ravassard P, Rousseau A, Boisselier B, Idbaih A, Calvo CF, Leuraud P, et al: ASPM-associated stem cell proliferation is involved in malignan progression of gliomas and constitutes an attractive therapeutic target. Cancer Cell Int 10: 1, 2010.

14. Peyre M, Commo F, Dantas-Barbosa C, Andreiuolo F, Puget S, Lacroix L, Drusch F, Scott V, Varlet P, Mauguen A, et al: Portrait of ependymoma recurrence in children: Biomarkers of tumor progression identified by dual-color microarray-based gene expression analysis. PLoS One 5: e12932, 2010.

15. Drozdov I, Bornschein J, Wex T, Valeyev NV, Tsoka S and Malfertheiner P: Functional and topological properties in hepatocellular carcinoma transcriptome. PLoS One 7: e35510, 2012.

16. Jung HM, Choi SJ and Kim JK: Expression profiles of SV40-immortalization-associated genes upregulated in various human cancers. J Cell Biochem 106: 703-713, 2009.

17. Kabbarah O, Nogueira C, Feng B, Nazarian RM, Bosenberg M, $\mathrm{Wu} \mathrm{M}$, Scott KL, Kwong LN, Xiao Y, Cordon-Cardo C, et al: Integrative genome comparison of primary and metastatic melanomas. PLoS One 5: e10770, 2010.
18. Brüning-Richardson A, Bond J, Alsiary R, Richardson J, Cairns DA, McCormack L, Hutson R, Burns P, Wilkinson N, Hall GD, et al: ASPM and microcephalin expression in epithelial ovarian cancer correlates with tumour grade and survival. $\mathrm{Br} \mathrm{J}$ Cancer 104: 1602-1610, 2011.

19. Wang WY, Hsu CC, Wang TY, Li CR, Hou YC, Chu JM, Lee CT, Liu MS, Su JJ, Jian KY, et al: A gene expression signature of epithelial tubulogenesis and a role for ASPM in pancreatic tumor progression. Gastroenterology 145: 1110-1120, 2013.

20. Xie JJ, Zhuo YJ, Zheng Y, Mo RJ, Liu ZZ, Li BW, Cai ZD, Zhu XJ, Liang YX, He HC and Zhong WD: High expression of ASPM correlates with tumor progression and predicts poor outcome in patients with prostate cancer. Int Urol Nephrol 49: 817-823, 2017.

21. Kim WJ, Kim EJ, Kim SK, Kim YJ, Ha YS, Jeong P, Kim MJ, Yun SJ, Lee KM, Moon SK, et al: Predictive value of progression-related gene classifier in primary non-muscle invasive bladder cancer. Mol Cancer 9: 3, 2010.

22. Riester M, Taylor JM, Feifer A, Koppie T, Rosenberg JE, Downey RJ, Bochner BH and Michor F: Combination of a novel gene expression signature with a clinical nomogram improves the prediction of survival in high-risk bladder cancer. Clin Cancer Res 18: 1323-1333, 2012.

23. El Behi M, Krumeich S, Lodillinsky C, Kamoun A, Tibaldi L, Sugano G, De Reynies A, Chapeaublanc E, Laplanche A, Lebret T, et al: An essential role for decorin in bladder cancer invasiveness. EMBO Mol Med 5: 1835-1851, 2013.

24. Hedegaard J, Lamy P, Nordentoft I, Algaba F, Høyer S, Ulhøi BP, Vang S, Reinert T, Hermann GG, Mogensen K, et al: Comprehensive transcriptional analysis of early-stage urothelial carcinoma. Cancer Cell 30: 27-42, 2016.

25. Subramanian A, Tamayo P, Mootha VK, Mukherjee S, Ebert BL, Gillette MA, Paulovich A, Pomeroy SL, Golub TR, Lander ES and Mesirov JP: Gene set enrichment analysis: A knowledge-based approach for interpreting genome-wide expression profiles. Proc Natl Acad Sci USA 102: 15545-15550, 2005. 